\title{
VARIATIONAL MULTISCALE METHOD WITH FLEXIBLE FINE- SCALE BASIS FOR DIFFUSION-REACTION EQUATION: BULT-IN A POSTERIORI ERROR ESTIMATE AND HETEROGENOUS COEFFICIENTS
}

\author{
MARCELINO ANGUIANO' ${ }^{1}$ AND ARIF MASUD ${ }^{2}$ \\ ${ }^{1}$ University of Illinois at Urbana-Champaign \\ 3103 Newmark Civil Eng. Bldg, 205 N Mathews Ave, Urbana, IL, USA 61801 \\ manguin2@illinois.edu \\ ${ }^{2}$ University of Illinois at Urbana Champaign \\ 3129E Newmark Civil Eng. Bldg, 205 N Mathews Ave, Urbana, IL, USA 61801 \\ amasud@illinois.edu, http://amasud.web.engr.illinois.edu/
}

Key words: Variational Multiscale Methods, Diffusion-Reaction Equation, Error Estimation, Heterogeneous Coefficients, Checkerboard Problem.

\begin{abstract}
The diffusion-reaction equation develops sharp boundary and/or internal layers for the reaction-dominated case (i.e. singularly perturbed case). In this regime, spurious oscillations pollute the solution obtained with the Galerkin finite element method (FEM). To address this issue, we employ a stabilized Variational Multiscale (VMS) method that relies on an improved expression for the fine-scale stabilization parameter that is derived via the fine-scale variational formulation facilitated by the VMS framework. The flexible fine scale basis consists of enrichment functions which may be nonzero at element edges. The stabilization parameter thus derived has spatial variation over element interiors and along inter-element boundaries that helps model the rapid variation of the residual of the Euler-Lagrange equations over the domain. This feature facilitates consistent stabilization across boundary and internal layers as well as capturing anisotropic refinement effects. In addition, VMS methods come equipped with useful a posteriori error estimators. New numerical results are presented that show the performance of this VMS method with a flexible fine-scale basis for singularly perturbed diffusion-reaction equation. These include an evaluation of the built-in error estimate for homogenous domain, and an optional modification of the method for heterogeneous domains that may result in savings in the computational cost.
\end{abstract}

\section{INTRODUCTION}

The Galerkin Finite Element method develops spurious oscillations engendered by boundary and/or internal layers in singularly perturbed diffusion-reaction equations (i.e., when the coefficient of the zeroth-order term is much larger than the coefficient of the second order term). An analysis that shows the origin of these spurious oscillations can be found in [1,2], and several methods have also been proposed to mitigate this issue [1,3-9]. 
The Variational Multiscale framework proposed by Hughes [6], generalized to bubble functions approach that was applied directly at the fine-scale variational level in Masud and colleagues [10-12], and formalized for hierarchical application of bubble functions approach in Masud and colleagues [13,14], was employed to derive a VMS method for singularly-perturbed diffusion-reaction equation in [15]. The key feature of the method in [15] is a more flexible fine-scale basis that consists of multiple local polynomial functions that may be nonzero on element boundaries. Sub-scale models that do not vanish at element boundaries also appear elsewhere in the literature [16-19]. However, the approach in [15] is distinct in that the element boundary terms are assumed to be negligible and interelement continuity of the fine-scales is not explicitly enforced. The resulting fine-scale model has increased flexibility in that it adapts locally and automatically to the restrictions and directionality imposed by the mesh and the internal and/or boundary layers. Furthermore, the method is more effective in satisfying the maximum principle, i.e., the spurious oscillations around boundary and internal layers are suppressed in most cases, or their magnitude is substantially reduced in comparison to other methods.

An additional feature of VMS methods is that they come equipped a posteriori error estimators [20]. In this work, we employ the VMS method from [15] to solve the singularly perturbed diffusion-reaction equation in homogeneous and heterogeneous domains. We present numerical results for the built-in error estimate for homogenous domain, as well as a possible modification of the method for heterogeneous domains that may yield computational cost
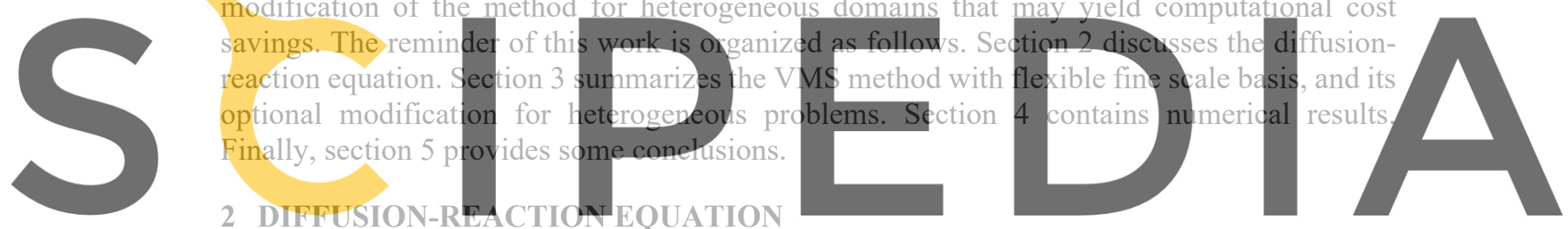

Register for free at https denote the boundary of the domain by $\partial \Omega$. Let $u: \Omega \rightarrow \mathbb{R}$ denote an unknown scalar field in

the domain. The reaction-diffusion equation with Dirichlet boundary condition is given by

$$
\begin{aligned}
-\varepsilon \Delta u+\sigma u & =f & & \text { in } \Omega \\
u & =g & & \text { on } \partial \Omega
\end{aligned}
$$

where $\varepsilon: \Omega \rightarrow \mathbb{R}^{+}$is the diffusion coefficient, $\sigma: \Omega \rightarrow \mathbb{R}^{+}$is the reaction coefficient, $f: \Omega \rightarrow \mathbb{R}$ is the forcing function, and $g$ is the prescribed Dirichlet boundary condition. We define the linear operator $\mathcal{L}:=-\varepsilon \Delta+\sigma$, so equation (1) can be written as

$$
\mathcal{L} u=f \quad \text { in } \Omega
$$

We further define the appropriate spaces of functions

$$
\begin{aligned}
& \mathcal{S}:=\left\{u \mid u \in H^{1}(\Omega), u=g \text { on } \partial \Omega\right\} \\
& \mathcal{V}:=\left\{w \mid w \in H^{1}(\Omega), w=0 \text { on } \partial \Omega\right\}
\end{aligned}
$$

and formulate the weak form of the problem: Given $\varepsilon, \sigma, f$, and $g$, find $u \in \mathcal{S}$ such that 


$$
(\nabla w, \varepsilon \nabla u)_{\Omega}+(w, \sigma u)_{\Omega}=(w, f)_{\Omega} \quad \forall w \in \mathcal{V}
$$

where $\left(v_{1}, v_{2}\right)_{\Omega}=\int_{\Omega} v_{1} \cdot v_{2} d \Omega$ denotes the $L^{2}$-inner product over the domain $\Omega$.

Numerical approximations to the solution of the weak form are formulated by the Galerkin method, where functions are taken from finite-dimensional subspaces of (4) and (5). The formal statement is: Given $\mathcal{E}, \sigma, f$, and $g$, find $u^{h} \in \mathcal{S}^{h} \subset \mathcal{S}$ such that

$$
\left(\nabla w^{h}, \varepsilon \nabla u^{h}\right)_{\Omega}+\left(w^{h}, \sigma u^{h}\right)_{\Omega}=\left(w^{h}, f\right)_{\Omega} \quad \forall w^{h} \in \mathcal{V}^{h} \subset \mathcal{V}
$$

where $\mathcal{S}^{h}$ and $\mathcal{V}^{h}$ are finite-dimensional subsets of the test and trial spaces respectively. In the context of the finite element method (FEM), these spaces consist of $C^{0}(\Omega)$ piecewise-

differentiable functions $C^{1}(K)$, commonly polynomials, with compact support defined over non-overlapping subdomains or elements, $K$. Rewriting equation (7), we obtain the following

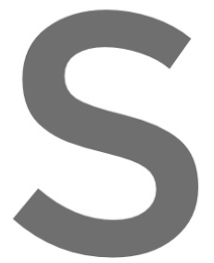

$$
\begin{array}{cc}
B_{\text {Gal }}\left(w^{h}, u^{h}\right)=L_{\text {Gal }}\left(w^{h}\right) & \forall w^{h} \in \mathcal{V}^{h} \subset \mathcal{V} \\
B_{\text {Gal }}\left(w^{h}, u^{h}\right)=\left(\nabla w^{h}, \varepsilon \nabla u^{h}\right)_{\Omega}+\left(w^{h}, \sigma u^{h}\right)_{\Omega}
\end{array}
$$

$$
L_{G a l}\left(w^{h}\right)=\left(w^{h}, f\right)_{\Omega}
$$
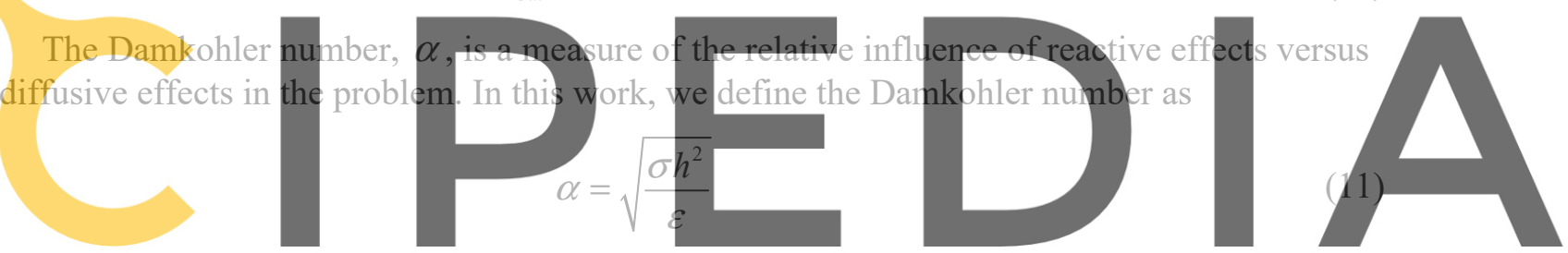

where $h$ is a certain length-scale associated with the discretized domain. In the context of the

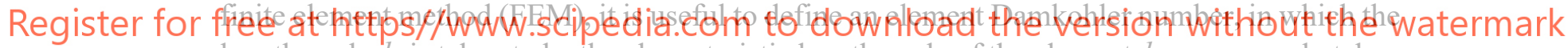
length-scale $h$ is taken to be the characteristic length-scale of the element $h_{K}$, commonly taken

as the element diameter or the element side length.

The Galerkin FEM exhibits spurious oscillations and the numerical solution fails to satisfy the maximum principle when the Damkohler number is larger than some critical value $\alpha_{c r}$. It has been shown in $[1,2]$ that $\alpha_{c r}=\sqrt{6}$ for the $1 \mathrm{D}$ case.

\section{VARIATIONAL MULTISCALE METHOD}

Stable numerical solution to the weak form is obtained by the VMS method. Here, we summarize the VMS method for singularly perturbed diffusion-reaction equation derived in [15], but recall that the consistent derivation of the method relies on additive decomposition of the solution $u$ into a coarse scale contribution $\bar{u}$ (associated with the standard finite element basis), and a fine scale contribution $u^{\prime}$ (associated with a fine-scale basis, linearly independent from the FE basis).

$$
u=\bar{u}+u^{\prime}
$$


The fine scales are defined in terms of a set of $n_{b}$ basis functions $B_{K}=\left\{b_{1}^{e}, b_{2}^{e}, \ldots, b_{n_{b}}^{e}\right\}$. For further details, the interested reader is referred to $[13,15]$. With these definitions, the resulting VMS method is stated as follows: Find $u^{h} \in \mathcal{S}^{h} \subset \mathcal{S}$ such that

$$
\begin{gathered}
B_{V M S}\left(w^{h}, u^{h}\right)=L_{V M S}\left(w^{h}\right) \quad \forall w^{h} \in \mathcal{V}^{h} \subset \mathcal{V} \\
B_{V M S}\left(w^{h}, u^{h}\right)=B_{G a l}\left(w^{h}, u^{h}\right)-\sum_{K}\left(\left(-\varepsilon \Delta w^{h}+\sigma w^{h}\right), \tau^{e}\left(-\varepsilon \Delta u^{h}+\sigma u^{h}\right)\right)_{K} \\
L_{V M S}\left(w^{h}\right)=L_{G a l}\left(w^{h}\right)-\sum_{K}\left(\left(-\varepsilon \Delta w^{h}+\sigma w^{h}\right), \tau^{e} f\right)_{K}
\end{gathered}
$$

where

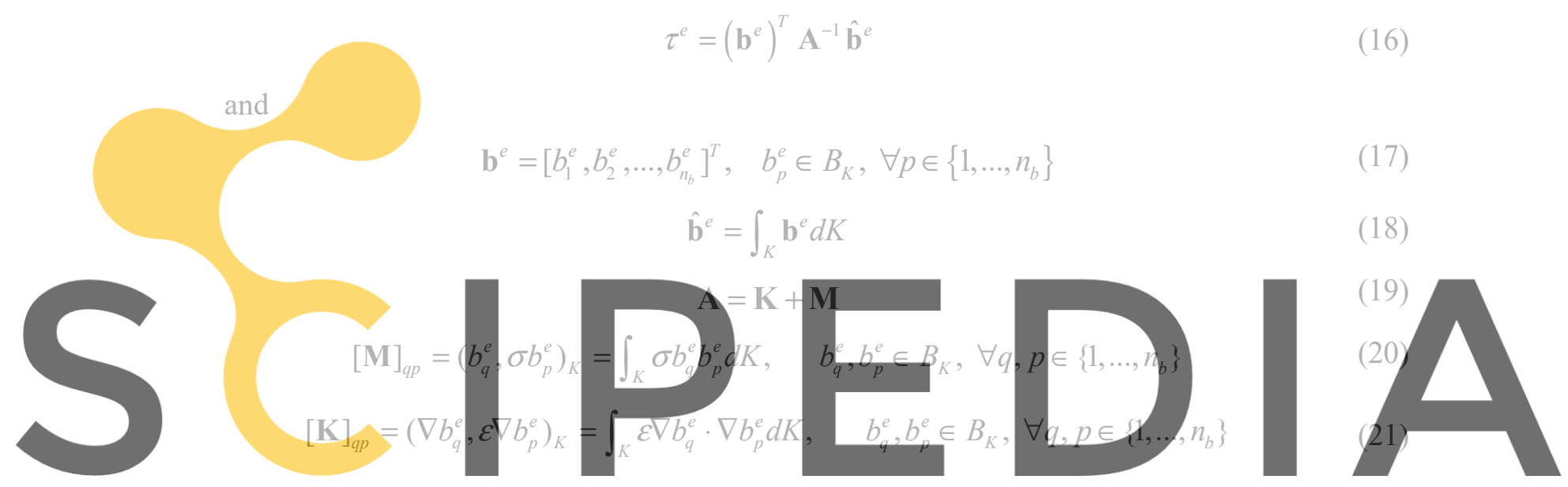

In earlier work by Masud and collaborators [21-24], a single bubble that is zero at the
In element boundaries was used to represent the fine scales, including some applications to reactive phenomena $[25,26]$. The example of a biquadratic bubble typically used with 4-node quadrilaterals is shown in Figure 1a. In this work we consider a fine-scale basis comprised of multiple functions that are not necessarily zero at the element boundary and are not necessarily enforced to be continuous across element interfaces. This amounts to a fine-scale representation with increased flexibility to be able to capture solutions that exhibit steep gradients, as in the case of the singularly perturbed (i.e., reaction-dominated) diffusion-reaction equation.

For 2D bilinear elements (Q4), the basis for the fine scales will be taken to be the shape functions from the Q9 element corresponding to nodes 5, 6, 7, 8, and 9, as sequenced in the element natural coordinates, shown in Table $1^{1}$. There, the first 4 functions correspond to the 4 edge functions (so-called "edge bubbles" used in [19]) and have the generic shape shown in Figure 1b, and the fifth function corresponds to the quadratic bubble shown in Figure 1a. We denote the resulting VMS method with multiple fine-scale basis functions as VMSm.

\footnotetext{
${ }^{1}$ For a more general discussion regarding the choice of the fine scale basis, the interested reader is referred to [15].
} 
(a)

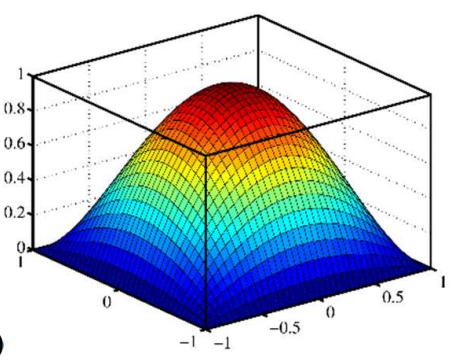

(b)

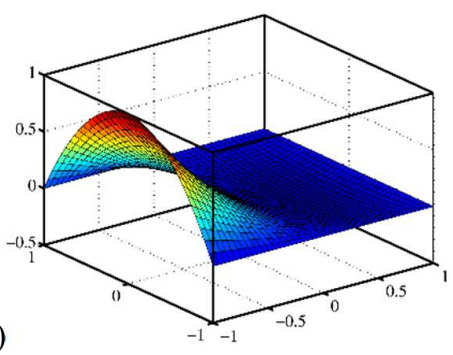

(c)

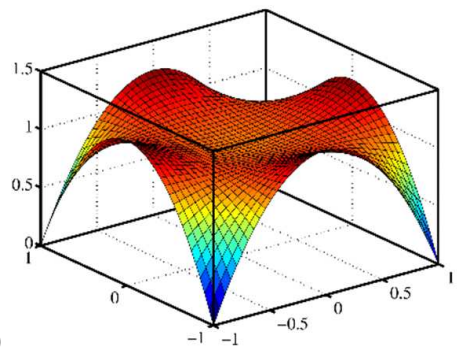

Figure 1: For Q4 element: (a) standard quadratic bubble, (b) quadratic edge function, and (c) example of their linear combination.

Table 1: Basis for the fine scales in the Q4 element (in element natural coordinates)

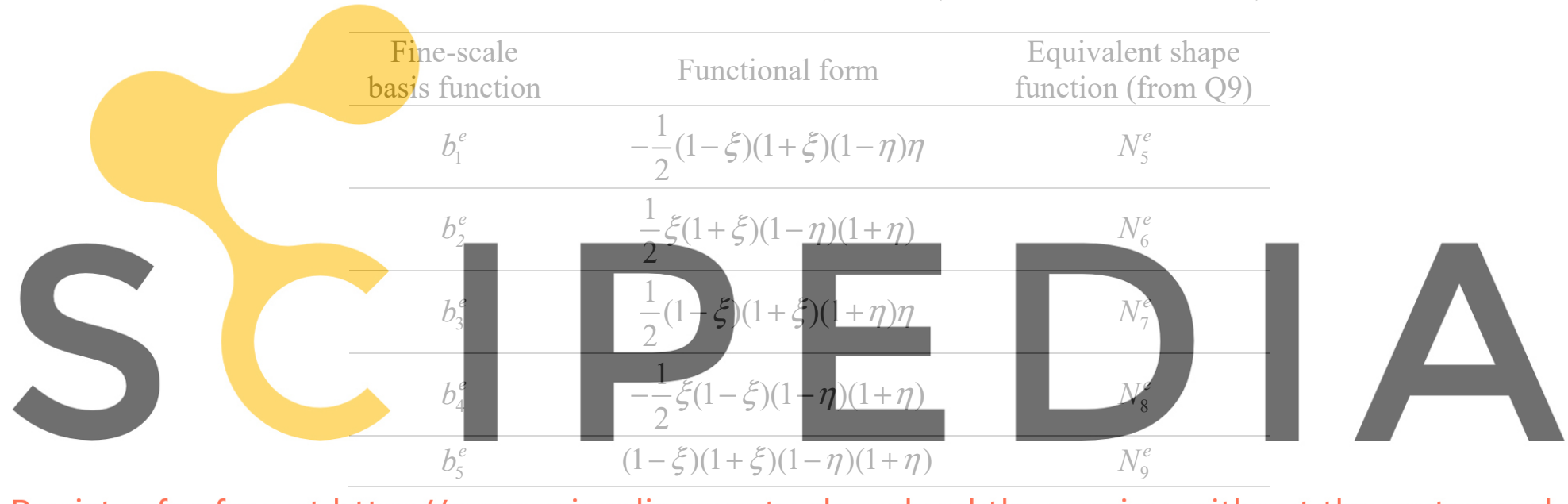

Register for free at https//www.scipedia.com to download the version without the watermark

\subsection{Built-in A Posteriori Error Estimate}

A feature of VMS methods is that they come equipped with an error estimation framework, as discussed in [20]. To see this, consider the multiscale decomposition of the exact solution in equation (12), where the fine scale field $u^{\prime}$ can be interpreted as the error $e$ between the exact solution $u$ and the coarse scale field $\bar{u}$

$$
e=u-\bar{u}=u^{\prime}
$$

In the derivation of VMS methods, the weak form is decomposed into a coarse scale subproblem and a fine scale subproblem. Solution of the coarse scale subproblem to find the coarse scale field depends on the fine scales, therefore $\bar{u}=\hat{u}\left(u^{\prime}\right)$. Invariably, in order to develop a numerical method, it is required to make approximations regarding the fine scales. In the present method, the fine scales are approximated locally within each element $K$ as

$$
\left.\left.u^{\prime}\right|_{K} \approx \tilde{u}^{\prime}\right|_{K}=\left.\tau^{e} \bar{R}\right|_{K}
$$

where $\bar{R}=\mathcal{L} \bar{u}-f=-\varepsilon \Delta \bar{u}+\sigma \bar{u}-f$ is the residual of the coarse scale Euler-Lagrange 
equations, and $\tau^{e}$ is given by (16).

The numerical solution $u^{h}$ to (13) represents an approximation to the exact coarse scales, i.e., $\bar{u} \approx u^{h}$, because of two factors: (i) in deriving the modified coarse scale problem in [15], some boundary terms were neglected, and (ii) the fine scale model is an approximation of the exact fine scales. Therefore, the total error in the numerical solution $e^{h}=u-u^{h}$ can be split into a local error $e_{L}$ associated with the error between the exact and approximated fine scales, and a global pollution error $e_{G}$. Moreover, the local error can be further split into an explicit term (given by the modeled fine scales) and an implicit correction. Therefore, the total error estimate is given as

$$
e^{h}=\hat{e}_{L}+\tilde{e}_{L}+\tilde{e}_{G}
$$

where the explicit error corresponds to the computed fine-scales.

$$
\left.\hat{e}_{L}\right|_{K}=\left.\tilde{u}^{\prime}\right|_{K}=\left.\tau^{e} \bar{R}\right|_{K}
$$

For detaits on the computation of the implicit parts of the a posteriori error estimation $\left(\tilde{e}_{L}\right.$ and $\left.\tilde{e}_{G}\right)$, the interested reader is referred to [20].
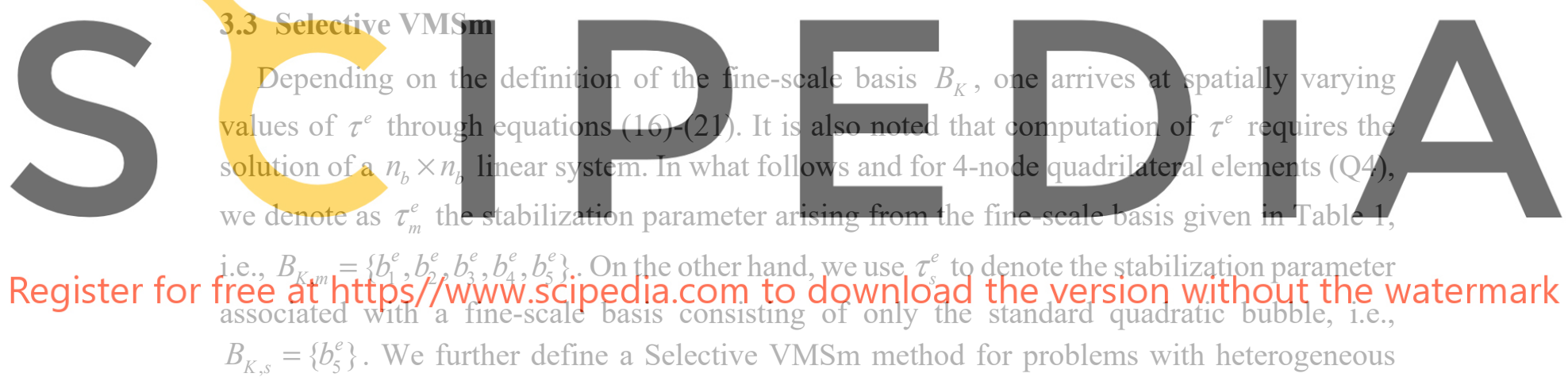

coefficients. In this method, the stabilization parameter $\tau^{e}$ within a given element $K$ is determined according to the value of the Damkohler number in that element $\alpha_{K}$ as follows

$$
\tau^{e}= \begin{cases}\tau_{m}^{e}, & \alpha_{K} \geq \alpha_{c r} \\ \tau_{s}^{e} . & \alpha_{K}<\alpha_{c r}\end{cases}
$$

The purpose of the definition in (26) is to reserve the use of the more flexible (but more computationally expensive) fine scale basis in regions of the domain where steep gradients are expected (i.e., reaction dominated regions with large Damkohler number), while using a single standard bubble in the diffusion dominated parts of the domain, for computational cost savings.

\section{NUMERICAL RESULTS}

In this section we present 2D numerical results for VMSm method applied to the scalar diffusion-reaction equation. First, we consider a problem with constant coefficients as presented 
in [15] and study the resulting fine-scale field as a built-in a posteriori error estimator, as discussed in Masud et al [20]. Secondly, we apply the VMSm and Selective VMSm method to solve the diffusion-reaction equation with heterogeneous coefficients with strong discontinuities in a checkerboard pattern.

\subsection{Error Estimate}

We discretize the unit square domain $\Omega=[0,1] \times[0,1]$ using a structured mesh of $20 \times 204$ node quadrilateral (Q4) elements. Then, we take the element side length as the characteristic length of the element $h_{K}=1 / 20=0.05$. The forcing function is set to $f=1$, the reaction coefficient is $\sigma=1$, and the diffusivity is $\varepsilon=1 e-6$, which results in a corresponding Damkohler number $\alpha=50>\alpha_{c r}$. Figure 2 shows results obtained usig the Galerkin FEM and

VMS with multiple fine-scale basis functions (VMSm). The Galerkin FEM solution exhibits large spurious oscillations around the boundary layers, while the solution obtained with the VMSm method captures the boundary layers sharply (within a single element) with no overshoots or undershoots.
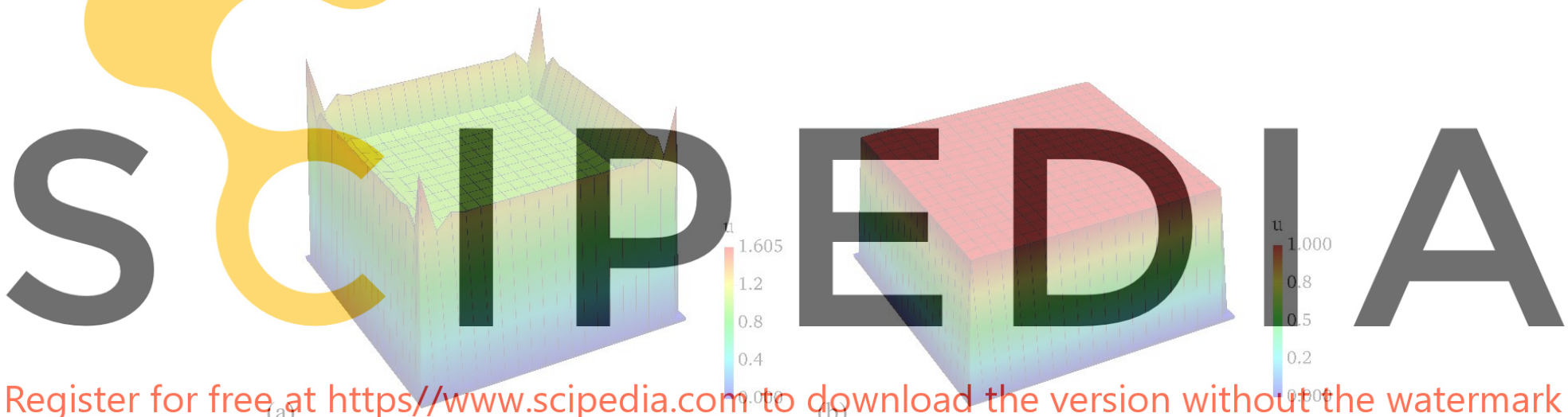

Register for free at https/wwww.scipedia.com oto dpwnload the version withoutethe watermark

Figure 2: Distribution of the local explicit error (or numerical fine scale field) in (a) the entire domain and (b) close-up of the top-right comer of the domain.

(a)

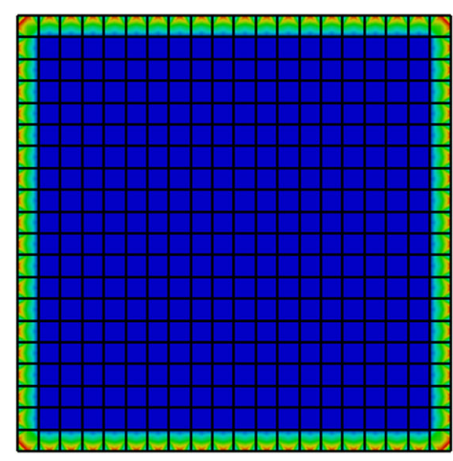

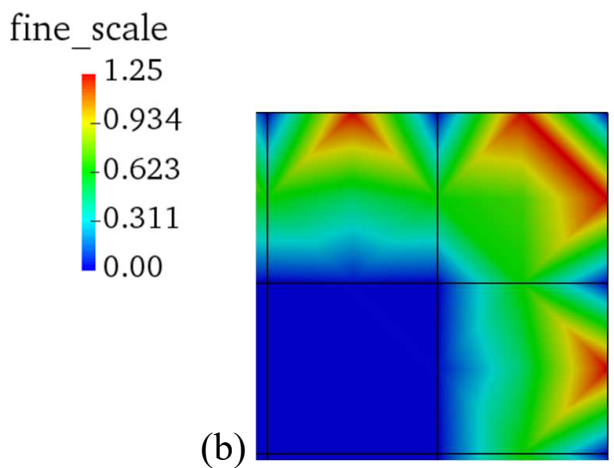

(b)

Figure 3: Distribution of the local explicit error (or numerical fine scale field) in (a) the entire domain and (b) close-up of the top-right corner of the domain. 
As discussed in section 3.2, the fine-scale field itself represents an error estimate. In particular, the numerical fine scales (expanded in terms of the fine scale basis) represent the socalled local explicit error $\hat{e}_{L}$ in the notation of [20]. Therefore, Figure 3 shows the distribution of the compute fine scales (or the local explicit error $\hat{e}_{L}$ ) in the domain. It is noted that this error estimate coincides with the notion that the largest errors occur in the boundary layer region. Furthermore, one may follow the framework provided in [20] to compute additional more accurate implicit estimates of local and global error, as well as compute the norms or seminorms (in $H^{1}(\Omega)$ or $L^{2}(\Omega)$ ) of these errors to verify their convergence. Nevertheless, we remark that the explicit local error already provides a reasonable estimate for the distribution of error in the domain.

\subsection{Heterogeneous Coefficients: Checkerboard Problem}

In this problem, we consider the scalar diffusion reaction equation with heterogeneous coefficients, i.e., $\sigma=\sigma(\mathbf{x})$ and $\varepsilon=\varepsilon(\mathbf{x})$. Moreover, there are strong discontinuities in the spatial variation of these material parameters as in equations (27) and (28), in a checkerboard pattern (Figure 4). The problem domain is the unit square $\Omega=[0,1] \times[0,1]$, and we impose homogeneous Dirichlet boundary conditions (Figure 4) and a constant forcing function $f=1$
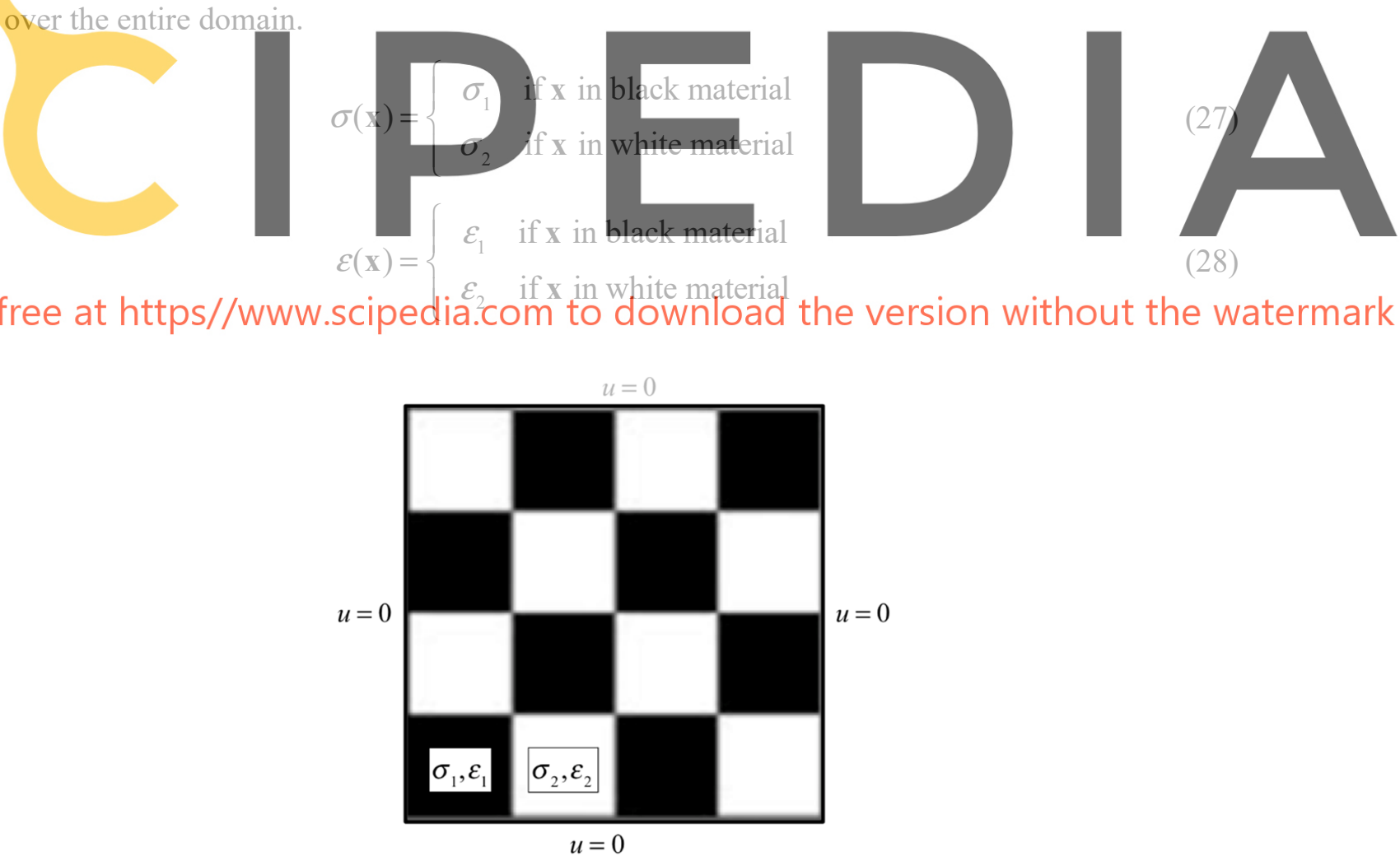

Figure 4: Schematic drawing of the domain for the checkerboard problem. 
The domain is discretized using a $20 \times 20$ grid of 4-noded quadrilateral elements. Then, the element length scale is $h_{K}=1 / 20=0.05$, and the Damkohler number for each material region in the checkerboard can be defined as in equation (29).

$$
\alpha_{1}^{2}=\frac{\sigma_{1} h_{K}^{2}}{\varepsilon_{1}}, \quad \text { and } \quad \alpha_{2}^{2}=\frac{\sigma_{2} h_{K}^{2}}{\varepsilon_{2}}
$$

The values of the coefficients are $\sigma_{1}=0.5, \varepsilon_{1}=1 \mathrm{e}-6, \sigma_{2}=1$, and $\varepsilon_{2}=1 \mathrm{e}-3$; consequently, the values of the Damkohler numbers are $\alpha_{1}^{2}=1250>\alpha_{c r}^{2}$ and $\alpha_{2}^{2}=2.5<\alpha_{c r}^{2}$. Thus, the black regions are reaction-dominated and the white regions are diffusion-dominated. The numerical results are shown in Figure 5 for different methods.

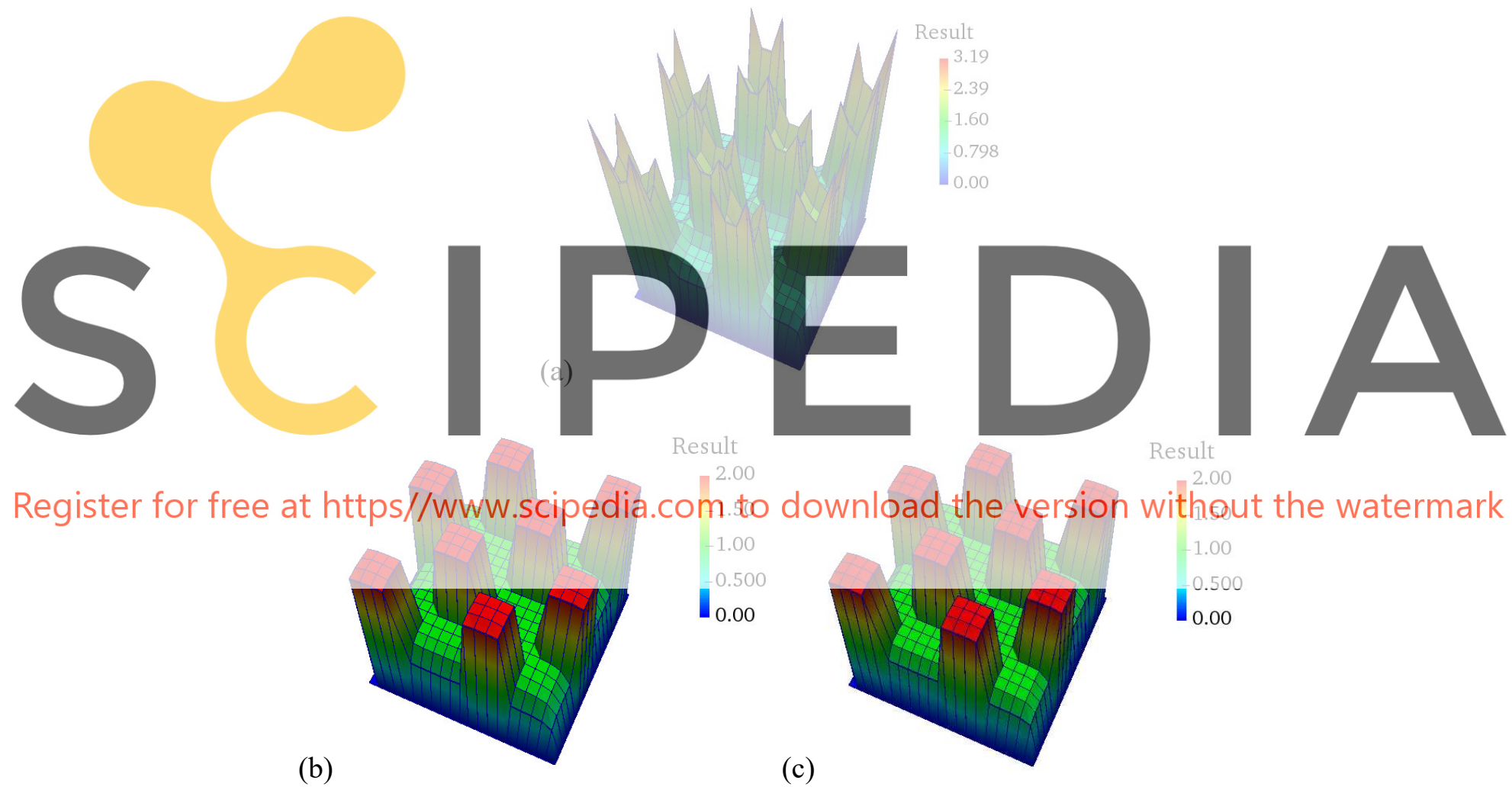

Figure 5: Plot of the solution field obtained with different methods: (a) Galerkin, (b) VMSm, and (c) Selective VMSm

The Galerkin solution shows spurious oscillations near the edges of the black squares of the checkerboard patten. These oscillations are suppressed by the VMSm and Selective VMSm methods. Furthermore, we remark that the maximum principle requires the exact solution to satisfy $u \leq \max f / \sigma=2$, which the Galerkin FEM solution does not, while the VMS solutions with flexible fine scale basis (VMSm and Selective VMSm) do satisfy it. Therefore, the VMSm 
method is robust for application to heterogeneous material properties, even with strong discontinuities in the spatial distribution of material coefficients.

For this case, computation of $\tau_{m}^{e}$ at the element level requires solution of a $5 \times 5$ system of linear equations, whereas computation of $\tau_{s}^{e}$ requires solution of a single linear equation. In practice, the additional cost incurred by solving the $5 \times 5$ systems in each element is negligible compared to other parts of the solution procedure (e.g., assembly, global problem solution). However, in higher dimensions and for higher order elements, the fine-scale basis may require more functions, and solution of the fine-scale problem may no longer be insignificant. In those situations, one may adopt the Selective VMSm approach to reduce computational cost.

\section{CONCLUSIONS}

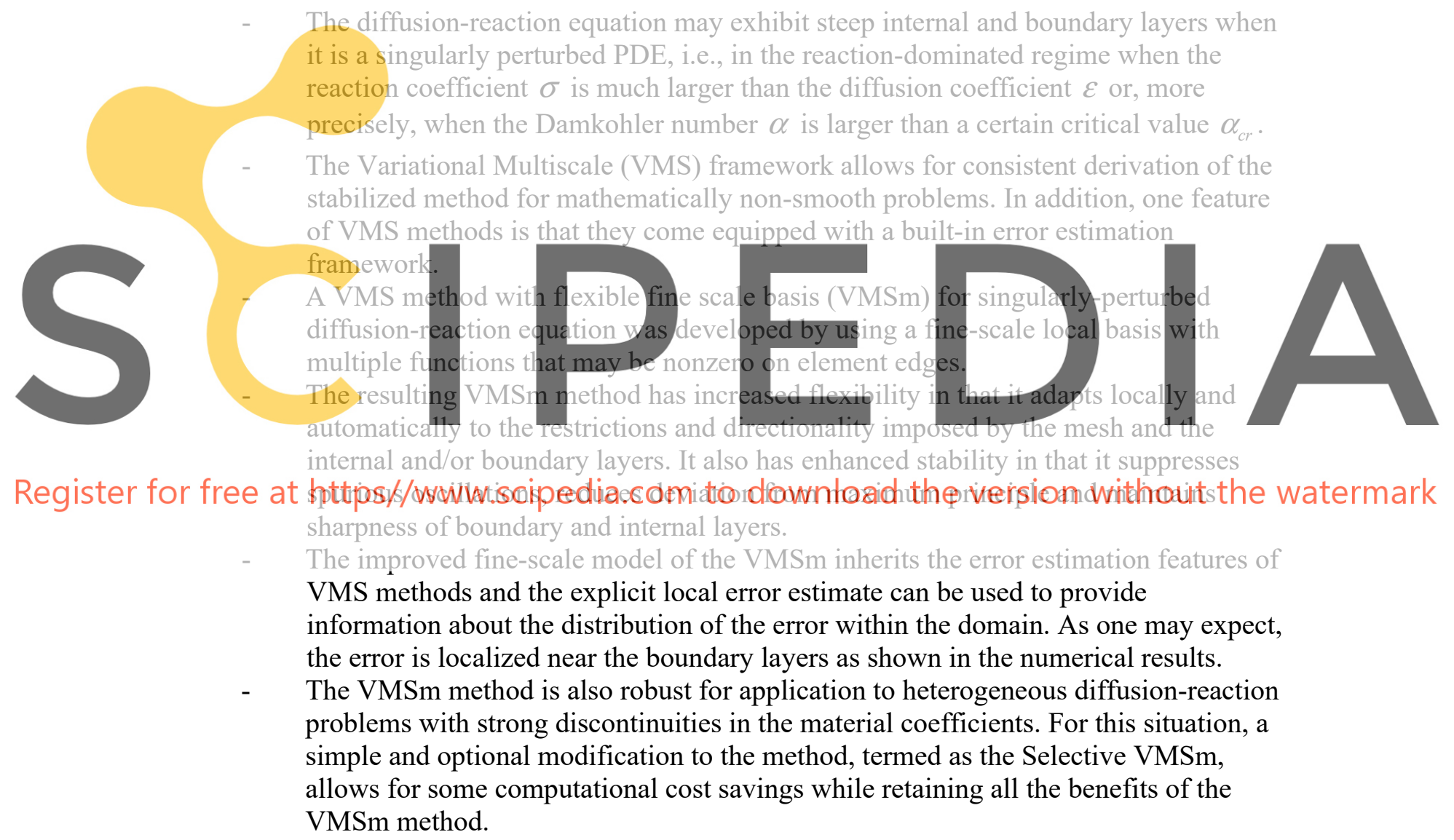

\section{REFERENCES}

[1] L.P. Franca, E.G. Dutra Do Carmo, The Galerkin gradient least-squares method, Comput. Methods Appl. Mech. Eng. 74 (1989) 41-54.

[2] I. Harari, Stability of semidiscrete formulations for parabolic problems at small time steps, Comput. Methods Appl. Mech. Eng. 193 (2004) 1491-1516. 
[3] T.E. Tezduyar, Y.J. Park, Discontinuity-capturing finite element formulations for nonlinear convection-diffusion-reaction equations, Comput. Methods Appl. Mech. Eng. 59 (1986) 307-325.

[4] I. Harari, T.J.R. Hughes, Stabilized finite element methods for steady advectiondiffusion with production, Comput. Methods Appl. Mech. Eng. 115 (1994) 165-191.

[5] L.P. Franca, C. Farhat, Bubble functions prompt unusual stabilized finite element methods, Comput. Methods Appl. Mech. Eng. 123 (1995) 299-308.

[6] T.J.R. Hughes, G.R. Feijóo, L. Mazzei, J.-B. Quincy, The variational multiscale method - a paradigm for computational mechanics, Comput. Methods Appl. Mech. Eng. 166 (1998) 3-24.

[7] E.G. Dutra do Carmo, G.B. Alvarez, F.A. Rochinha, A.F.D. Loula, Galerkin projected residual method applied to diffusion-reaction problems, Comput. Methods Appl. Mech. Eng. 197 (2008) 4559-4570.

[8] H. Fernando, C. Harder, D. Paredes, F. Valentin, Numerical multiscale methods for a reaction-dominated model, Comput. Methods Appl. Mech. Eng. 201-204 (2012) 228244.

[9] E. Oñate, P. Nadukandi, J. Miquel, Accurate FIC-FEM formulation for the multidimensional steady-state advection-diffusion-absorption equation, Comput. Methods Appl. Mech. Eng. 327 (2017) 352-368.

[10] M Ayub, A Masud, A new stabilized formulation for convective-diffusive heat transfer, Numerical Heat Transfer: Part B: Fundamentals 44 (1) (2003) 1-23.

[11] A. Masud, R.A. Khurram, A multiscale/stabilized finite element method for the advection-diffusion equation, Comput. Methods Appl. Mech. Eng. 193 (2004) 19972018.

[12] A Masud, JH Kwack, A stabilized mixed finite element method for the first-order form of advection-diffusion equation, International journal for numerical methods in fluids 57 (9), (2008) 1321-1348.

[13] A Masud, LP Franca, A hierarchical multiscale framework for problems with multiscale source terms, Computer Methods in Applied Mechanics and Engineering 197 (33-40), (2008) 2692-2700.

[14] A Masud, G Scovazzi, A heterogeneous multiscale modeling framework for hierarchical systems of partial differential equations, International journal for numerical methods in fluids 65 (1-3), (2011) 28-42.

[15] A. Masud, M. Anguiano, I. Harari, Modeling of steep layers in singularly perturbed diffusion-reaction equation via flexible fine-scale basis, Comput. Methods Appl. Mech. Eng. 372 (2020) 113343.

[16] C. Farhat, I. Harari, L.P. Franca, The discontinuous enrichment method, Comput. Methods Appl. Mech. Eng. 190 (2001) 6455-6479.

[17] N.C.B. Arruda, R.C. Almeida, E.G. Dutra do Carmo, Discontinuous subgrid formulations for transport problems, Comput. Methods Appl. Mech. Eng. 199 (2010) 3227-3236.

[18] C. Coley, J.A. Evans, Variational multiscale modeling with discontinuous subscales: analysis and application to scalar transport, Meccanica. 53 (2018) 1241-1269.

[19] T.J. Truster, A. Masud, Primal interface formulation for coupling multiple PDEs: A consistent derivation via the Variational Multiscale method, Comput. Methods Appl. 
Mech. Eng. 268 (2014) 194-224.

[20] A. Masud, T.J. Truster, L.A. Bergman, A variational multiscale a posteriori error estimation method for mixed form of nearly incompressible elasticity, Comput. Methods Appl. Mech. Eng. 200 (2011) 3453-3481.

[21] A. Masud, R. Calderer, A variational multiscale method for incompressible turbulent flows: Bubble functions and fine scale fields, Comput. Methods Appl. Mech. Eng. 200 (2011) 2577-2593.

[22] A Masud, TJ Truster, LA Bergman, A unified formulation for interface coupling and frictional contact modeling with embedded error estimation, International journal for numerical methods in engineering 92 (2), (2012) 141-177.

[23] A. Masud, T.J. Truster, A framework for residual-based stabilization of incompressible finite elasticity: Stabilized formulations and F bar methods for linear triangles and tetrahedra, Comput. Methods Appl. Mech. Eng. 267 (2013) 359-399.

[24] Finite strain primal interface formulation with consistently evolving stabilization, TJ Truster, P Chen, A Masud, International Journal for Numerical Methods in Engineering 102 (3-4), (2015) 278-315.

[25] M. Anguiano, H. Gajendran, R.B. Hall, K.R. Rajagopal, A. Masud, Chemo-mechanical coupling and material evolution in finitely deforming solids with advancing fronts of reactive fluids, Acta Mech. 231 (2020) 1933-1961.

[26] R. Hall, H. Gajendran, A. Masud, Diffusion of chemically reacting fluids through nonlinear elastic solids: mixture model and stabilized methods, Math. Mech. Solids. 20 (2015) 204-227. 